\title{
Coherent wavepackets in the Fenna-Matthews- Olson complex are robust to excitonic-structure perturbations caused by mutagenesis
}

\author{
Margherita Maiuri ${ }^{*}$, Evgeny E. Ostroumov ${ }^{1}$, Raphael G. Saer ${ }^{2}$, Robert E. Blankenship ${ }^{2}$ \\ and Gregory Scholes ${ }^{1}$ \\ ${ }^{1}$ Department of Chemistry, Princeton University, Princeton, New Jersey 08544, USA \\ ${ }^{2}$ Departments of Biology and Chemistry, Washington University, St. Louis, Missouri, USA
}

\begin{abstract}
The photosynthetic FMO complex is probed by femtosecond pump-probe spectroscopy and compared with a series of genetically modified mutants with distinct excitonic interactions. These experiments allow us to identify vibrational contributions to the coherence.
\end{abstract}

Nature has developed sophisticated ways to control photosynthetic complexes where the arrangement of the individual pigments is engineered to funnel the absorbed energy and transfer it to reaction centres with evolutionary efficiency ( $>95 \%$ ) [1]. In the last decade, it has been discovered that light-harvesting in Nature may involve quantum-coherence [2]. The early observation of long-lived coherent oscillations at cryogenic and ambient temperatures in the photosynthetic complexes of a green sulfur bacteria, the FennaMatthews-Olson (FMO) complex, has generated deep interest in the role of such beatings in energy transfer [3]. Theoretical studies have now predicted that the coherent oscillations observed in many ultrafast experiments are assigned to vibronic coherence, a quantum mechanical mixture of vibrational levels and excited electronic states [4].

Here we study the coherent oscillations in FMO complex by exploiting the combination of femtosecond spectroscopy and biological mutagenesis [5]. We report a pump-probe investigation of a series of FMO complexes, where site-mutagenesis enabled us to perturb the energies of selected chromophores, thereby changing their electronic interactions, and compare the generated coherences. This approach allowed us to probe the contributions of specific excitons to the coherent beatings. Through a systematic comparison of the series of mutated FMO complexes measured under two excitation conditions and at two temperatures, we show that the strongest oscillations detected in our experiment are insensitive to mutagenesis, having similar frequencies and dephasing times in all of the pump-probe data. We also show that in the selective pump-probe experiments the oscillations are due to the ground state vibrational wavepackets. Additionally, the selective pump-probe experiments reveal significant interchromophore coupling, which is observed throughout the absorption spectrum of the FMO complexes.

The arrangement of the BChls in the FMO complex is depicted in Fig. 1a, together with its cryogenic absorption spectrum which reveals three distinct $\mathrm{Q}_{\mathrm{y}}$ (lowest lying electronic state of a BChl) bands (fig. 1b). The spectral positions of these bands are primarily determined by the interaction of the BChls with the charged amino acids and ligands and by 
electronic coupling among different $\mathrm{BChl}$ molecules, which results in seven delocalized excitonic states. Fig. 1 also reports the two BChls (BChl 6 and BChl 3) that have been perturbed and the corresponding spectra of the mutated FMO complexes (W184F and Y16F) [6]. The absorption spectrum of the W184F mutant reveals major changes at the $\mathrm{Q}_{\mathrm{y}}$ transitions in the high energy side, while the Y16F exhibits a dramatic decrease in the amplitude of the lowest energy band.
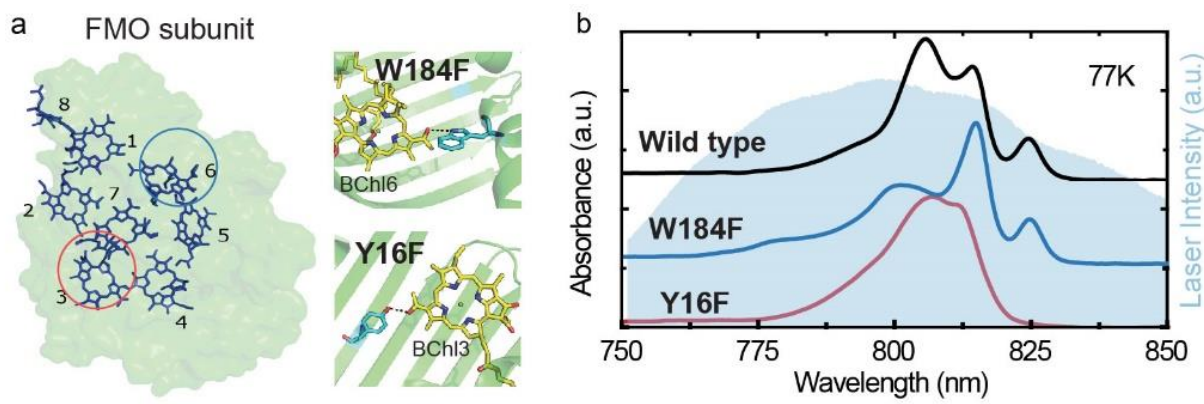

Fig. 1. (a) Crystal structure of the FMO subunit and highlights the perturbed BChls in the mutants. (b) Absorption spectra for the FMO and the two mutant complexes at $77 \mathrm{~K}$.

Figure 2a shows the results of the broad-band pump-probe experiments performed at $77 \mathrm{~K}$ on the FMO wild type. To investigate coherent oscillations, the exponential decay contribution has been removed from the pump-probe maps and the residual oscillatory traces have been analyzed both in time and frequency domains. Figs. $2 b$ report residual oscillation traces extracted from the $77 \mathrm{~K}$ data of the wild type at three selected probe wavelengths. We observe distinguishable beating signals lasting more than $2 \mathrm{ps}$ in all the traces, which were fit by two damped cosine functions (black solid lines in Figure 2b). The Fourier transformed power spectra of both the experimental and simulated data are shown in Fig. 2c and compared with the Fourier Spectra obtained using the same procedure for the two mutants (2d-e). The two observed oscillation frequencies (ca. $160 \mathrm{~cm}^{-1}$ and $190 \mathrm{~cm}^{-1}$ ) are very similar across the spectrum for all the samples.

To further examine the coherent wavepackets we performed another series of pumpprobe experiments where the excitation spectrum was narrowed and tuned to the red edge of the FMO absorption spectrum (fig. 2f). Upon excitation of the red shoulder of the FMO absorption spectrum, we detect coherent oscillations at all probe wavelengths, even on the blue edge of the probe spectrum where no direct pump excitation takes place. The two main oscillation frequencies agree with those of the broad-band experiment and are observed in the selective experiments of the all FMO complexes (fig. 2h-j). 

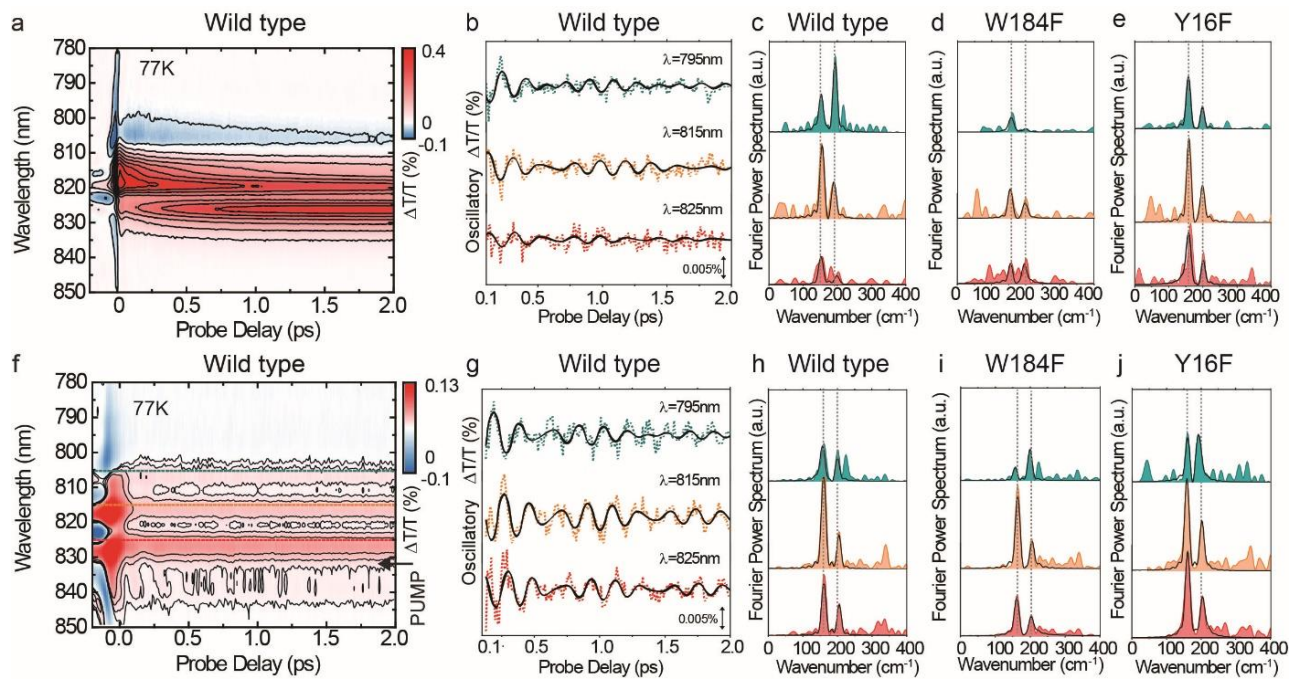

Fig. 2. Pump-probe map at $77 \mathrm{~K}$ for the wild type FMO complex measured upon (a) broadband pump excitation and (f) selective red-edge excitation. (b) Selected residual oscillations obtained after removing a two-exponential decay component from the pump-probe data in (a). (c) Corresponding Fourier spectra obtained from (b) for the wild type and for W184F (d) and Y16F (e) obtained at the same probe wavelength. (g) Selected residual oscillations obtained after removing a two-exponential decay component from the pump-probe data in (f). (h) Corresponding Fourier spectra obtained from (g) for the wild type and for $\mathrm{W} 184 \mathrm{~F}$ (i) and $\mathrm{Y} 16 \mathrm{~F}$ (j) obtained at the same probe wavelength

Our series of experiments show that: (i) the main oscillations dephasing on a picosecond time scale are surprisingly insensitive to the spectral mutations. (ii) By studying these coherences with selective excitation of the lowest excitonic state, we assign them to ground state vibrational oscillations. Our study reveals that coherent wavepackets in the transient absorption spectra of FMO complexes comprise ground-state vibrations, which are observed in our data via coherently delocalized exciton states.

This discovery contributes to the discussion about the influence of vibronic coupling in light-harvesting and how artificial systems should be designed.

\section{Reference}

1. R. E. Blankenship Molecular mechanisms of photosynthesis (Blackwell Science Ltd, 2002)

G. S. Engel, T. R. Calhoun, E. L. Read, T. K. Ahn, T. Mančal, Y. C. Cheng, R. E. Blankenship, G. R. Fleming, Nature 446, 782-786 (2007)

2. G. Panitchayangkoon, D. Hayes, K. A. Fransted, J. R. Caram, E. Harel, J. Wen, R. E. Blankenship, G. S. Engel, PNAS 107, 12766-12770 (2010).

3. N. Christensson, H. F. Kauffmann, T. Pullerits, and T. Mancal, J Phys Chem B 116, 7449-7454 (2012)

4. M. Maiuri, E. E. Ostroumov, R. G. Saer, R. E. Blankenship, G. D. Scholes, Nature Chemistry 10 177-183 (2018)

5. R. G. Saer, V. Stadnytskyi, N. C. Magdaong, C. Goodson, S. Savikhin, R. E. Blankenship, Biochimica et Biophysica Acta 1858, 288-296 (2017) 